\title{
Evaluation of the Effects of Aluminum Phosphate and Calcium Phosphate Nanoparticles as Adjuvants in Vaccinated Mice
}

\author{
Aliaa M. Issa, Mohamed S. Salim, Hamdallah Zidan, Aly F. Mohamed, and Abdel-Razik H. Farrag
}

\begin{abstract}
The present study aim at studying the histological effects of both aluminum phosphate (Alum) and calcium phosphate (CAP) nanoparticles adjuvant in parallel with their potentials as adjuvant and the related immune response to tetanus toxoid vaccine adsorbed on both of them. Ninety Swiss albino mice were used in the experiment $(50 \%$ adults and $50 \%$ juveniles). Mice were immunized intramuscularly with $0.125 \mathrm{ml}$ adjuvanted tetanus toxoid vaccine. For alum adjuvant study, 27 adult mice and 27 juvenile ones were injected with alum adjuvanted vaccine and sacrificed weekly as triplects for 9 weeks. For calcium phosphate adjuvant study, 15 adult mice and 15 juvenile ones were injected with calcium phosphate adjuvanted vaccine and sacrificed weekly as triplects for 5 weeks. The effect of alum and calcium phosphate nanoparticles adjuvants in enhancing the immune response of tetanus toxoid vaccine were monitored through measurement of antibody titer in sera of mice. The pathological effect of both adjuvants were monitored through histological study of liver, brain, kidney and injected muscle of sacrificed animals. Recorded data revealed that both adjuvanted vaccine caused histopathological changes in tissues of liver, kidney, brain and injected muscle. On the other hand alum adjuvanted tetanus toxoid vaccine was more potent and showed higher antibody level than CAP adjuvanted vaccines.
\end{abstract}

Index Terms-Aluminum phosphate, calcium phosphate, nanoparticles, adjuvant, histology, vaccine.

\section{INTRODUCTION}

Vaccines usually require additional exogenous adjuvants to improve the immune response to the antigens following immunization [1]. Hence, one of the most significant challenges in vaccinology is the selection of suitable adjuvants [2]. Virtually, all adjuvant systems developed to date have focused on one of two mechanisms: specific immune activation or the delivery-depot effect [3]. Although many adjuvant systems have been developed and tested in preclinical models, few have actually proved useful for human vaccines. The primary limitations for the use of new adjuvant systems with human vaccines revolve around safety issues [3]. They added also that the toxicity

Manuscript received November 10, 2013; revised February 7, 2014.

Aliaa M. Issa is with the Department of Zoology, Faculty of Science, Cairo University, Giza, Egypt (e-mail: aliaai@yahoo.com).

Mohamed S. Salim and Aly F. Mohamed are with the Holding Company for Biological Products and Vaccines (VACSERA), Dokki, Giza.

Hamdallah Zidan is with the Department of Microbiology and Immunology, Faculty of Pharmacy, Cairo University, Egypt.

Abdel-Razik H. Farrag is with the Department of Pathology, Medical Division, National Research Center, Dokki, Cairo, Egypt. of adjuvants has been reduced systematically through research and development efforts over the last 80 years. In the United States, alum compounds are the most extensively used adjuvants in licensed vaccines for humans, although they effectively enhance immune responses, there are several disadvantages associated with their use [4]. The disadvantages of alum-based adjuvants include the severity of local tissue irritation, the longer duration of the inflammatory reaction at the injection site, minimal induction of cell-mediated immunity and a propensity to elicit undesirable immunoglobulin $\mathrm{E}$ (IgE) responses [5]. For these reasons, new adjuvants are being developed to enhance the immunity against weak antigens. Nanomaterial has unique physicochemical properties, such as ultra-small size, large surface area to mass ratio, and high reactivity, which are different from bulk materials of the same composition. These properties can be used to overcome some of the limitations found in traditional vaccines [6]. Efforts with calcium adjuvants have continued, and work with calcium phosphate nanoparticles has had some preclinical success [7]. Calcium phosphate (CAP) and aluminium phosphate (alum) compounds have been approved as vaccine adjuvants for human use in several European countries [8]. This study aims at evaluating the effect of both aluminum phosphate and calcium phosphate nanoparticles used as vaccine adjuvants on the immune response and the histological structure of liver, brain, kidney and injected muscle of adult and juvenile immunized mice.

\section{MATERIALS AND METHODS}

\section{A. Animals}

Ninety Swiss albino mice were used in this experiment, obtained from the Egyptian Company for the Production of Sera and Vaccines (EgyVac.), affiliate of the Holding Company for Biological Products and Vaccines (Vacsera). Forty five of them were adult mice (18-22 gm) and the other forty five were juvenile (10-12 gm). The mice were housed under standard condition in plastic laboratory cages in the animal facility of the same place and maintained on a standard mouse diet of pellets and water ad-libitum. All animals used in the study were allowed to acclimatize for a period of one week before the start of the experiment.

\section{B. Preparation of Adjuvant}

Aluminum phosphate (Alum) nanoparticles adjuvant: Alum adjuvant was prepared according to the methods of [9], [10]. 
Calcium phosphate (CAP) nanoparticles adjuvant:

CAP nanoparticles adjuvant was prepared according to the method of [11].

\section{Immunization of the Experimental Laboratory Animals}

Mice were immunized intramuscularly with the equivalent dose which is the highest dose tolerated by mice $(0.125 \mathrm{ml})$ [12].

\section{Evaluation of Immune Response against Alum and Calcium Phosphate Adjuvanted Vaccine by Antibody Titer in Serum of Immunized Animals}

The antibody titer against immunization with vaccine adsorbed on both alum and CAP was evaluated using indirect enzyme linked immunosorbent assay (indirect ELISA) [12]. The immune response was detected in the sera of the sacrificed mice of all groups using indirect Eliza method [12], [13].

\section{E. Histological Study}

Small specimens from liver, kidney, brain and the injected muscle were collected from dissected mice, fixed in neutral buffered formalin then proceeded to obtain $5 \mu \mathrm{m}$ hematoxylin and eosin sections were prepared, examined with light microscope for the identification of the histological changes [14]. A number of photomicrographs were taken at known magnification using (Leica DMLS light microscope).

\section{F. Study Design}

Animals were divided into three main groups:

Group I (a \& b) Consisted of 3 adult and 3 juvenile mice (respectively) and sacrificed after the 1rst week.

Group II (a \& b) Consisted of 54 mice, divided into two groups, each of 27 mice/group, adult and juvenile mice (respectively). Immunized with alum adjuvanted tetanus toxoid vaccine and sacrificed after the $1^{\text {rst }}$ to the $9^{\text {th }}$ week post immunization.

Group III ( $\mathrm{a} \& \mathrm{~b}$ ) Consisted of a total of 30 mice, divided into 15 mice/group, adult and juvenile mice (respectively). Immunized with CAP adjuvanted tetanus toxoid vaccine and sacrificed after the 1 rst to the $5^{\text {th }}$ week post immunization.

Evaluation of antibody titer in serum of all sacrificed animals took place, and for histological study, the liver, brain, kidney and injected muscle were removed after animal sacrifice.

The experimental time durations were chosen according to [15] who reported that the irritation caused by an aluminum hydroxide gel and suspension persisted for 8 weeks, while the local tissue reactions caused by the injection of CAP gel and suspension completely ceased by the 4 th week. Thus, the present study continued for 9 weeks post immunization in case of alum adjuvanted vaccine and 5 weeks in case of CAP adjuvanted vaccine and the samples were collected weekly.

\section{G. Statistical Analysis}

Data were analyzed for statistical significance by a oneway analysis of variance (ANOVA). P value of $<0.05$ was considered as a significant [16].

\section{RESULTS}

\section{A. Evaluation of Immune Response Study}

The immune response was compared between adult and juvenile groups for each used adjuvant (alum and CAP). Another comparison was also conducted between the immune response against alum, CAP and positive control (immunized with tetanus toxoid only) in both adult and juvenile groups.

In case of the immunization with alum adjuvanted tetanus toxoid, the antibody level showed higher values in adult than juvenile groups along the 9 studied weeks except at the $6^{\text {th }}$ week post immunization where the antibody level of the juvenile group was higher than that of the adult ones (Fig. 1). In case of the immunization with CAP adjuvanted tetanus toxoid, the antibody level was higher in the adult groups than juvenile ones along the 5 weeks of the experiment except at the $4^{\text {th }}$ week post immunization where the antibody level was higher in the juvenile group than adult ones (Fig. 2).

Antibody production by adult and juvenile mice are shown in Fig. 1 and Fig. 2 respectively.

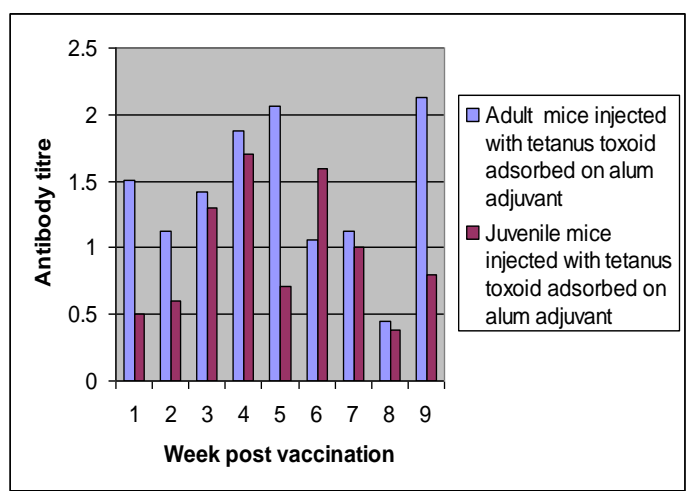

Fig. 1. Antibody production by adult and juvenile mice following immunization with tetanus toxoid adsorbed on alum adjuvant.

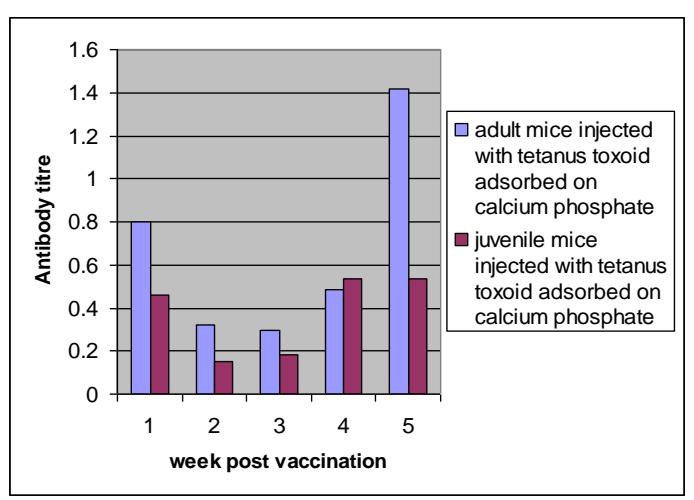

Fig. 2. Antibody production by adult and juvenile mice following immunization with tetanus toxoid adsorbed on calcium phosphate adjuvant.

The antibody production following the injection of adult and juvenile groups with tetanus toxoid only (as positive control), tetanus toxoid adsorbed on alum and tetanus toxoid adsorbed on CAP was compared. It was found that the antibody level recorded higher values along the whole experiment, when using alum adjuvant than when using CAP adjuvant, and already than the positive control (without using adjuvant). These results were found in both adult and juvenile groups (Fig. 3 \& Fig. 4) respectively. 


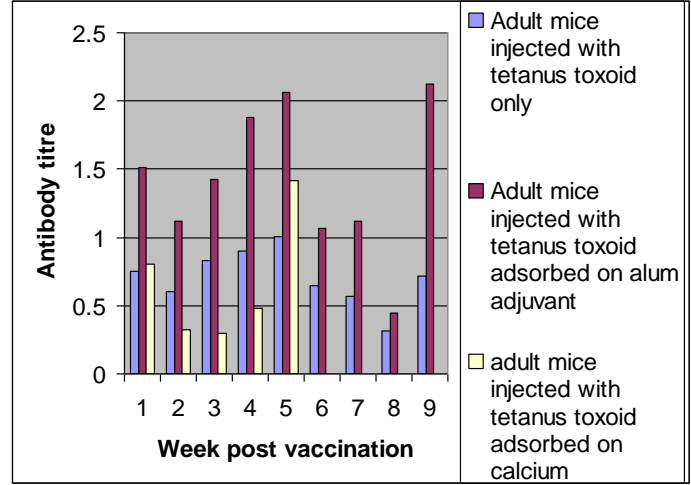

Fig. 3. Antibody production by adult mice following injection with tetanus toxoid only, tetanus toxoid adsorbed on alum and tetanus toxoid adsorbed on calcium phosphate.

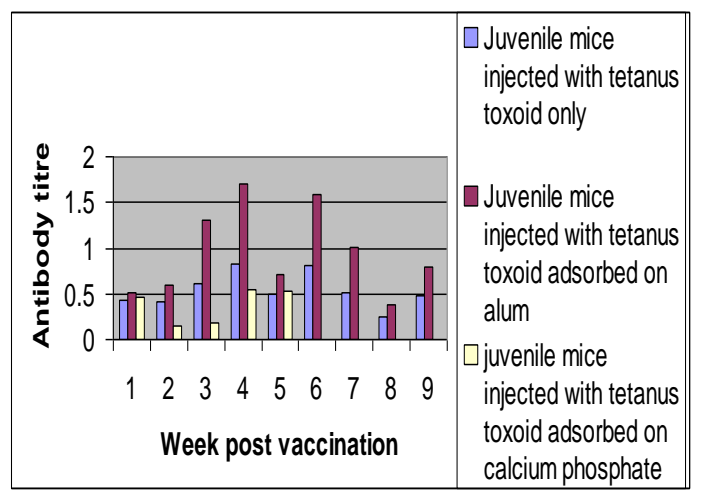

Fig. 4. Antibody production by juvenile mice following injection with tetanus toxoid only, tetanus toxoid adsorbed on alum and tetanus toxoid adsorbed on calcium phosphate.

\section{B. The Histological Study}

The results revealed that the histological changes in the liver of the adult and juvenile groups were severe in case of using tetanus toxoid adjuvanted on either alum or CAP nanoparticles. These changes included hepatocellular necrosis infiltrated by mononuclear cells, hydropic degeneration and distinct Küpffer cells (Fig. 5-Fig. 10).

The histological changes of the kidney were severe with the use of alum adjuvant in both adult and juvenile groups. This included mononuclear cell infiltration, vacuolation of renal tubule epithelium and congestion of peritubular capillaries. While the changes in case of CAP nanoparticles adjuvant,the changes were restricted to the appearance of mononuclear cell aggregation (Fig. 11-Fig. 13).

The histological changes observed in the brain were also severe in case of alum adjuvant with both adult and juvenile groups and were more obvious at the $6^{\text {th }}$ week post immunization and it included cerebral congestion, meningeal hemorrhage, thickening and hyalinization of blood vessel, neuronal degeneration and brain edema. While the changes in case of calcium phosphate included cerebral congestion and cerebral tissue vacuolation (Fig. 14-Fig. 16).

The histological changes occurring in the injected muscle were severe in case of CAP nanoparticle adjuvant with both adult and juvenile groups and it included large aggregation of mononuclear cells, intramuscular hemorrhage, and hyaline degeneration of muscle fiber, muscular necrosis and calcium deposition while the changes in case of alum adjuvant included mononuclear cell infiltration and muscular hemorrhage (Fig. 17-Fig. 21).

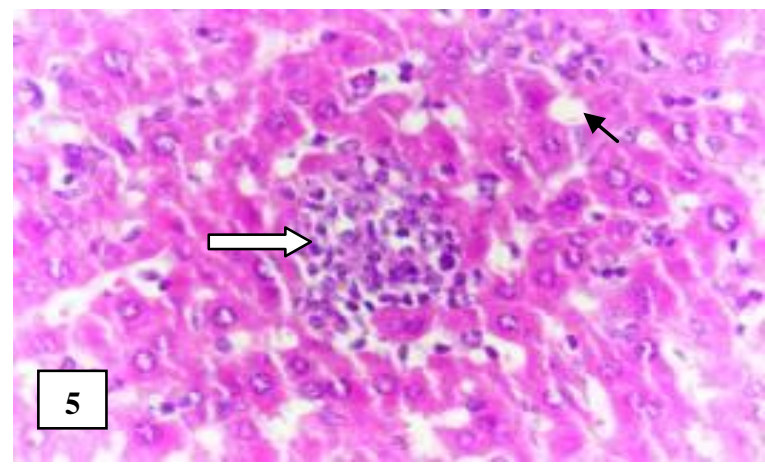

Fig. 5. Photomicrograph of liver section of adult mouse immunized with tetanus toxoid adsorbed on alum adjuvant 1 week post immunization showing hepatocellular necrosis (arrow) replaced by mononuclear cells (white arrow) (H \& E, X 400).

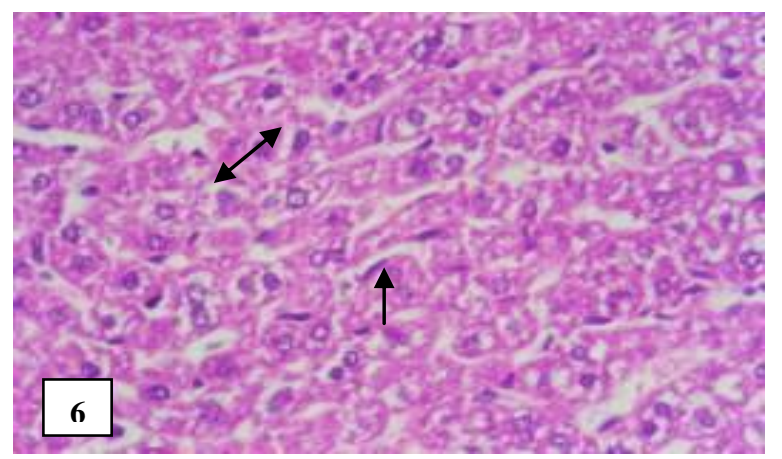

Fig. 6. Photomicrograph of liver section of juvenile mouse immunized with tetanus toxoid adsorbed on alum adjuvant 2 weeks post immunization showing individual hepatocellular necrosis (double head arrow) with Küpffer cell (arrow) (H \& E, X 400).

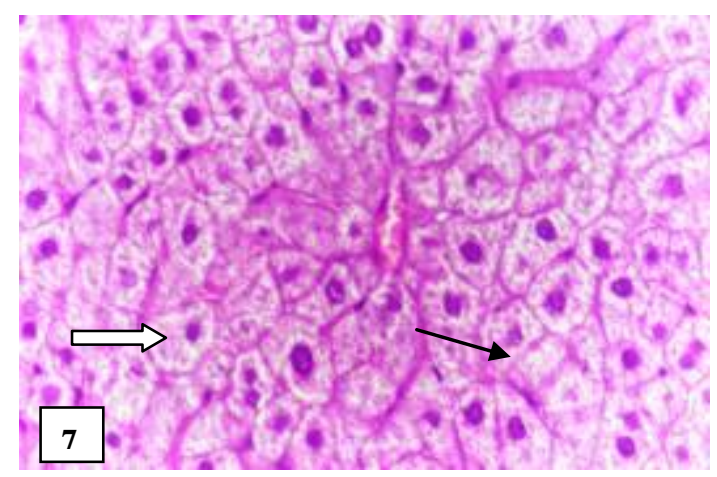

Fig. 7. Photomicrograph of liver section of juvenile mouse immunized with tetanus toxoid adsorbed on alum adjuvant 5 weeks post immunization showing hydropic degenerated cells with cytoplasmic swelling (white arrow) and individual cell necrosis (arrow) (H \& E, X 400).

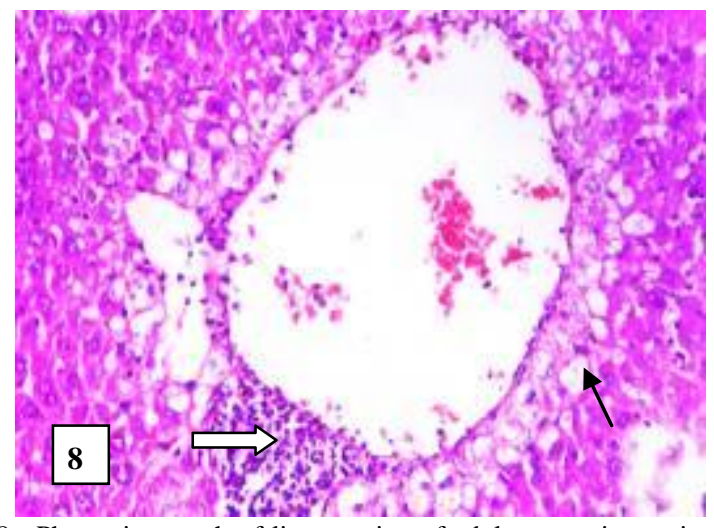

Fig. 8. Photomicrograph of liver section of adult mouse immunized with tetanus toxoid adsorbed on calcium phosphate nanoparticles adjuvant 1 week post immunization showing vacuolation (arrow) and necrosis of centrilobular hepatocytes with perivascular mononuclear cell aggregation (white arrow) (H \& E, X 200). 


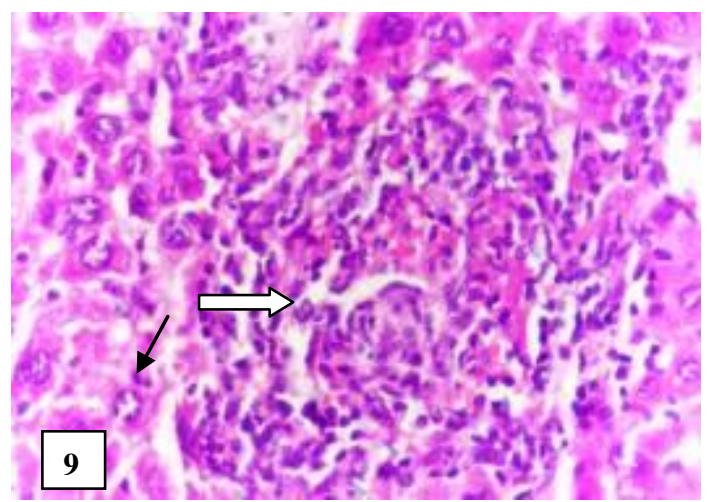

Fig. 9. Photomicrograph of liver section of adult mouse immunized with tetanus toxoid adsorbed on calcium phosphate nanoparticles adjuvant 1 week post immunization showing hepatocellular necrosis (arrow) infiltrated by mononuclear cells (white arrow) (H \& E, X 400).

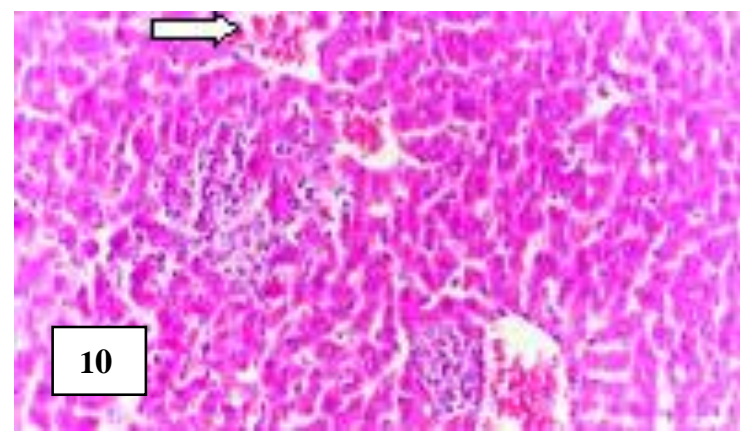

Fig. 10. Photomicrograph of liver section of juvenile mouse immunized with tetanus toxoid adsorbed on calcium phosphate nanoparticles adjuvant 1 week post immunization showing perivascular mononuclear cell aggregation (arrow) with infiltration of hepatic sinusoids (white arrow) $(\mathrm{H}$ \& E, X 200).

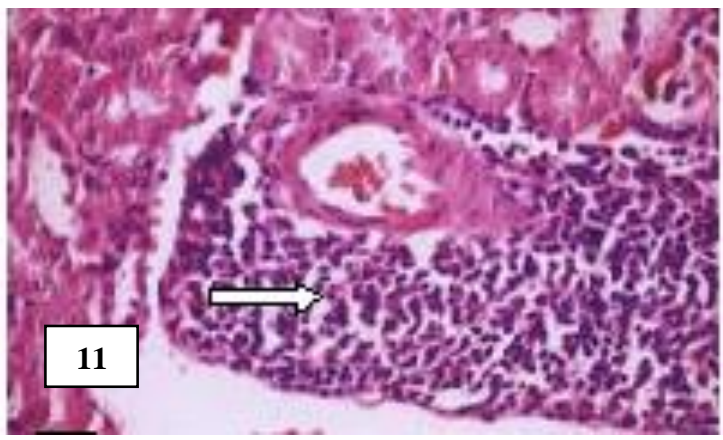

Fig. 11. Photomicrograph of kidney section of adult mouse immunized with tetanus toxoid adsorbed on calcium phosphate nanoparticles adjuvant 1 week post immunization showing intense perivascular aggregation of mononuclear cells (white arrow) (H \& E, X 400).

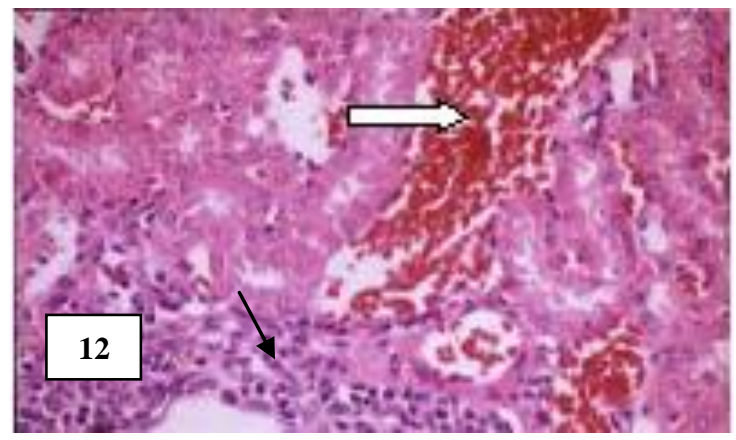

Fig. 12. Photomicrograph of kidney section of juvenile mouse immunized with tetanus toxoid adsorbed on alum adjuvant 1 week post immunization showing mononuclear cell infiltration (arrow) in interstitial tissue with congestion of peritubular capillaries (white arrow) (H \& E, X 400).

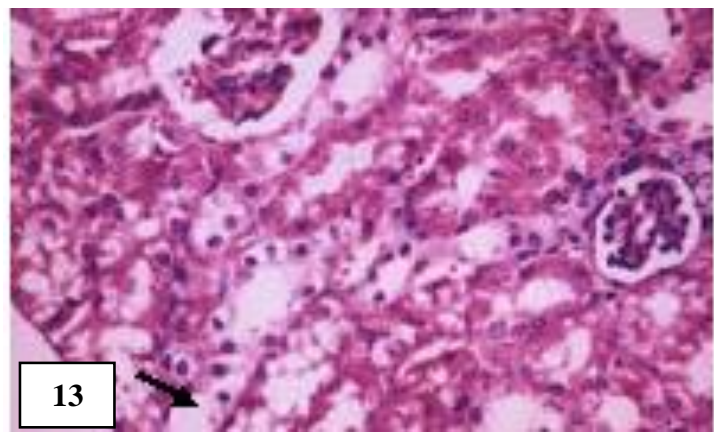

Fig. 13. Photomicrograph of kidney section of juvenile mouse immunized with tetanus toxoid adsorbed on alum adjuvant 2 weeks post immunization showing vacuolation of renal tubular epithelium (arrow) (H \& E, X 400).

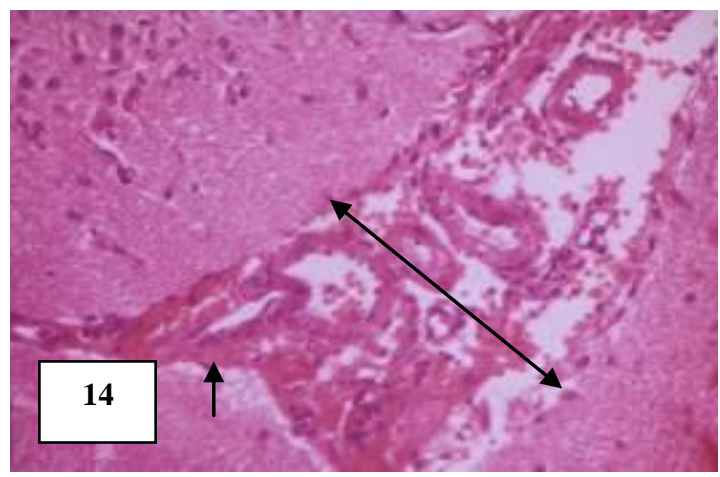

Fig. 14. Photomicrograph of brain section of adult mouse immunized with tetanus toxoid adsorbed on alum adjuvant 6 week post immunization showing thickening and hyalinization (double head arrow) of blood vessel wall associated with hemorrhage (arrow) (H \& E, X 200).

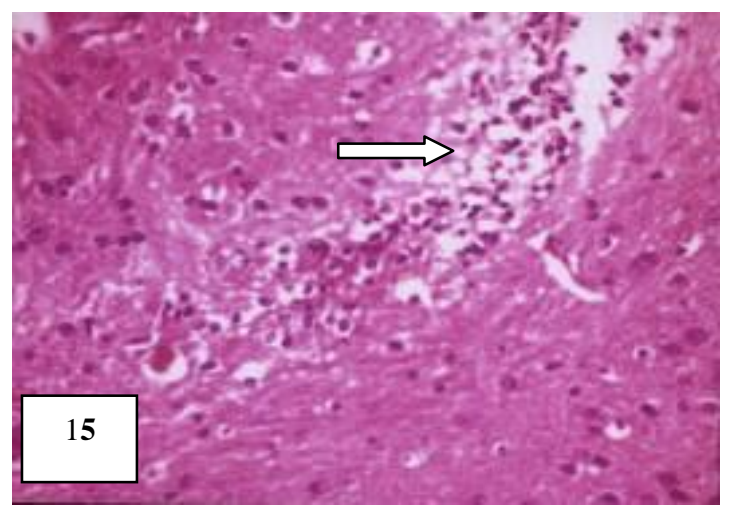

Fig. 15. Photomicrograph of brain section of adult mouse immunized with tetanus toxoid adsorbed on alum adjuvant 6 weeks post immunization showing brain edema (white arrow) (H \& E, X 400).

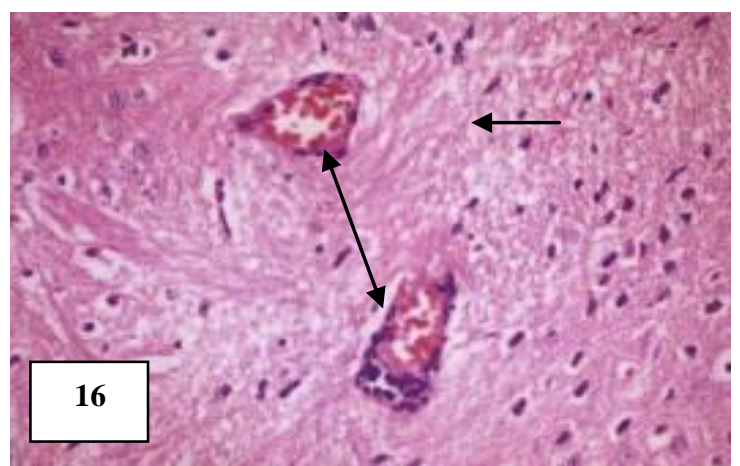

Fig. 16. Photomicrograph of brain section of adult mouse immunized with tetanus toxoid adsorbed on calcium phosphate nanoparticles adjuvant 1 week post immunization showing cerebral tissue vacuolation (arrow) and cerebral congestion (double head arrow) (H \& E, X 200). 


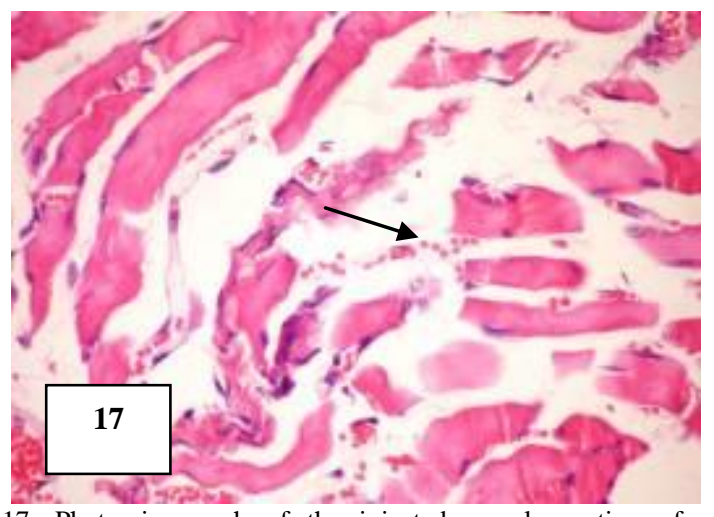

Fig. 17. Photomicrograph of the injected muscle section of mouse immunized with tetanus toxoid adsorbed on alum adjuvant 1 week post immunization showing muscular hemorrhage (arrow) (H \& E, X 400).

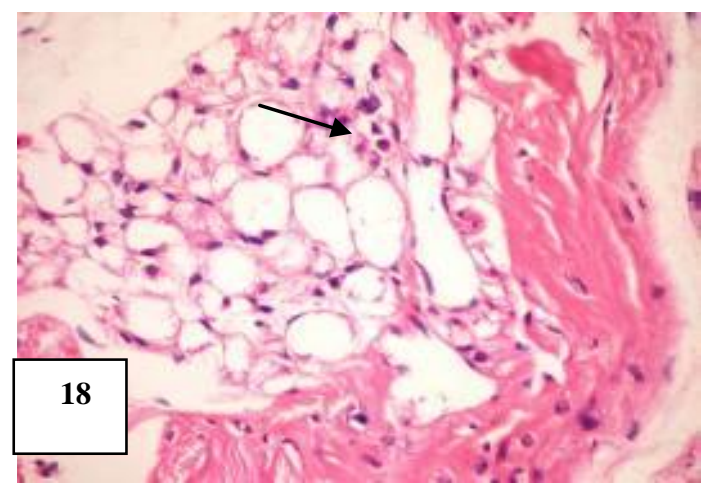

Fig. 18. Photomicrograph of the injected muscle section of mouse injected with tetanus toxoid adsorbed on alum adjuvant 1 week post immunization showing mild infiltration of mononuclear cells (arrow) in epimysium( $\mathrm{H} \&$ E, X 400).

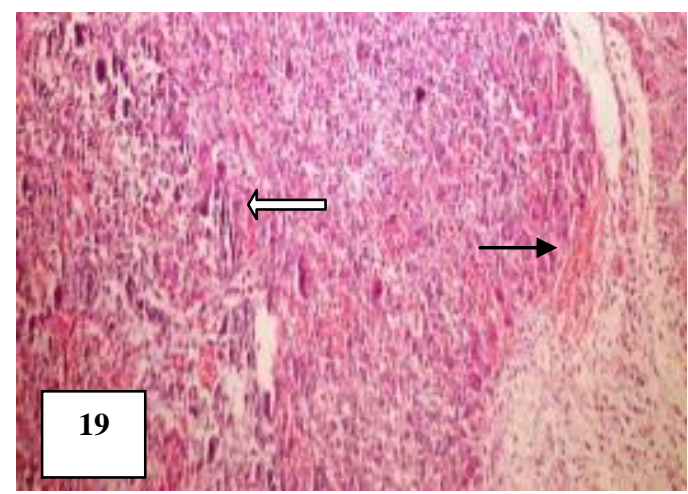

Fig. 19. Photomicrograph of the injected muscle section of adult mouse immunized with tetanus toxoid adsorbed on calcium phosphate nanoparticles adjuvant 1 week post immunization showing large aggregation of mononuclear cells (white arrow) with area of hemorrhage (arrow) replacing epimysium (H \& E, X 400).

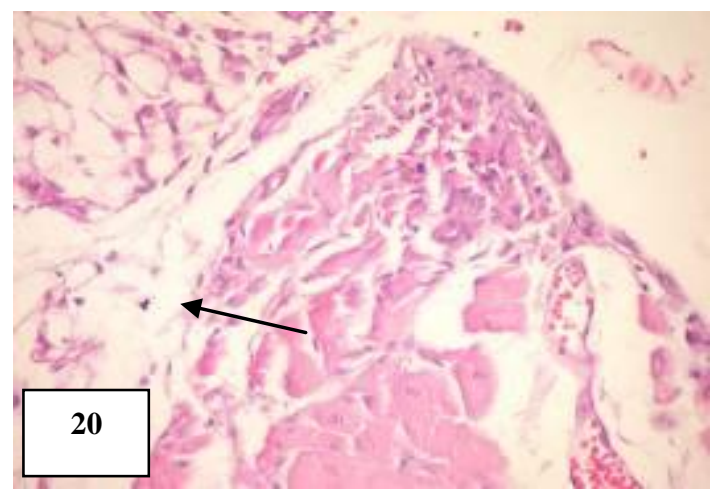

Fig. 20. Photomicrograph of the injected muscle section of juvenile mouse immunized with tetanus toxoid adsorbed on calcium phosphate nanoparticles adjuvant 1 week post immunization showing hyaline degeneration of muscle fibers (arrow) (H \& E, X 400).

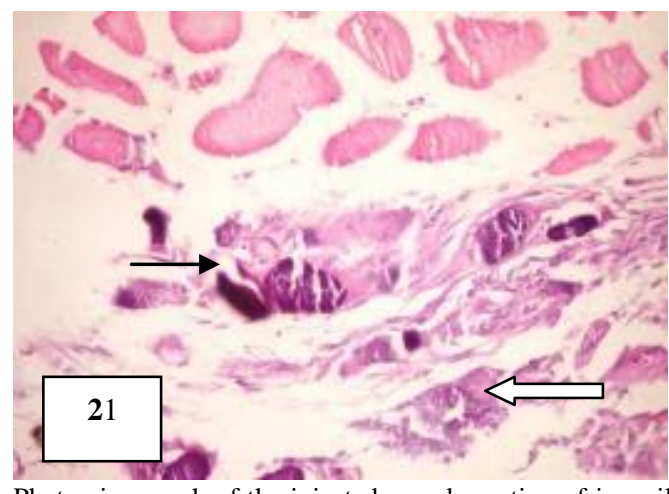

Fig. 21. Photomicrograph of the injected muscle section of juvenile mouse immunized with tetanus toxoid adsorbed on calcium phosphate nanoparticles adjuvant 1 week post immunization showing necrosis (white arrow) associated with calcium deposits (arrow) (H \& E, X 400).

\section{DISCUSSION}

Vaccines have profound impact on global health although concerns persist about their potential role in autoimmune or other adverse reactions [17]. To address these concerns, vaccine components like immunogens and adjuvants require critical evaluation for healthy subjects and their safety. In the present study, aluminium phosphate (alum) and calcium phosphate (CAP) nanoparticle were prepared and used as adjuvants.

The results revealed that the used adjuvants increased the immune response of the vaccine adsorbed on them. But it was higher in case of alum than in CAP nanoparticles. The immune response was also higher in adult mice than in juvenile ones in most results, which mean that it could be age dependent. This is in agreement with [17], [18] who stated that CAP adjuvant induced a lower level of IgE when compared to alum adjuvant and with [19], [20] who observed a decreased level of local irritation in experimental animals.

The histological changes in the liver of adult and juvenile groups were severe in case of both alum and CAP nanoparticles. These changes included hepatocellular necrosis infiltrated by mononuclear cells, hydropic degeneration and appearance of Küppfer cells. Küpffer cells activation might indicate that nanoparticles activate the phagocytic activity of the sinusoidal cells by increasing the number of Küpffer cells to help in removing the accumulated nanoparticles [21]. The swelling of hepatocytes might be exhibited as a result of disturbances of membranes function, that could be confirmed by the accompanied leakage of lysosomal hydrolytic enzymes [22]. The hydropic degeneration is a result of ion and fluid homeostasis unbalance that lead to an increase in intracellular water [22]. The histological changes appearing in the kidney of adult and juvenile groups were pronounced in case of alum adjuvant. The changes included mononuclear cell infiltration, vacuolation of renal tubule epithelium and congestion of peritubular capillaries, while the changes in case of CAP nanoparticles adjuvant were restricted to the appearance of mononuclear cell aggregation. Infiltration of monocytes to kidney is known to correlate with proteinuria and onset of kidney damage [23]. The histological changes observed in the brain of both adult and juvenile groups were well noticed in case of alum adjuvant and more obvious at the $6^{\text {th }}$ week 
post immunization. The changes included cerebral congestion, meningeal hemorrhage, thickening and hyalinization of blood vessel, neuronal degeneration and brain edema. These results were in agreement with the results of many studies [24], where mentioned that aluminum in particular has long been associated with neuronal degeneration and neurodegenerative diseases [25]. It was stated that aluminum adjuvanted vaccines showed an increase in aluminum levels in the murine brain [26]. They suggested that aluminum is transported to the brain by the iron-binding protein transferrin and enters the brain via specific transferrin receptors. On the other hand [27] work agreed with the present results of the alum severe effect on the brain suggesting that the enhancement of inflammation and the interference with cholinergic projections may be the modes of action through which aluminum may cause learning and memory deficits, and contribute to pathological processes in Alzheimer's disease. The changes in case of CAP nanoparticles adjuvant included cerebral congestion and cerebral tissue vacuolation, these changes appeared in both adult and juvenile groups. Ref. [28] stated that calcium phosphosilicate nanocomposite particles can be effectively targeted to gastrin receptors in vivo in a model of pancreatic cancer, and further showed the potential for targeting across the blood-brain-barrier. This could explain the potential of the CAP molecules to penetrate the blood-brain-barrier and causing the observed changes. It was also stated that because nanoparticles can pass through biological membranes, they can affect the physiology of most cells, including brain and testes [29]. CAP nanoparticles could induce increase of cells arrest in $S$, $\mathrm{S} /(\mathrm{G} 2 / \mathrm{M})$ and increased apoptotic population by interferencing mitochondrial structure and function [30].

The histological changes occurring in the injected muscle of both adult and juvenile groups in case of alum adjuvent included mononuclear cell infiltration and muscular hemorrhage are on line with [31], [32]. While in case of using CAP nanoparticles adjuvant the changes were severe. The changes included large aggregation of mononuclear cells, intramuscular hemorrhage, hyaline degeneration of the muscle fiber, muscular necrosis and calcium deposition. The results of [33] disagreed with the present work. They postulated that this was probably due to the fact that the CAP nanoparticles are not recognized by the inflammatory cells due to their smaller size. On the other hand the present study did not agree with what mentioned [34] that tissue reactions caused by injection of CAP adjuvant completely ceased by the 4 th week, while irritation caused by alum persisted till 8 weeks.

The results of the present work showed toxic effects on the different studied tissues (liver, kidneys, brain and the injected muscle). This could be explained on the basis that the small size of CAP with its higher permeability to cell membranes and consequence directly affected the cells leading to the mentioned pathological changes observed in the examined tissue [35]. The hemorrhages indicated that the endothelium was damaged due to the direct toxic effect of the used adjuvant, however; the presence of monocytic infiltration indicated that the body immune response dealt with this foreign substance as a toxic material [35].

\section{CONCLUSION}

The result of this study revealed that the presence of adjuvant has marked increase in antibody level meanwhile the presence of alum in vaccine models has better immune response than CAP nanoparticle where none adsorbed vaccines showed the lowest antibody level. At the same time both of used adjuvants has histological effects which mainly targeting the liver and some other organs.

\section{ACKNOWLEDGMENT}

The authors would also like to thank Dr. Faten F. Mohamed from the Department of Pathology, Faculty of Veterinary Medicine, Cairo University, Giza, Egypt for her valuable comments and cooperation in the description of the histological sections.

\section{REFERENCES}

[1] P. Marrack, A. S. McKee, and M. W. Munks, "Towards an understanding of the adjuvant action of aluminium," Nat Rev Immunol, vol. 9, pp. 287-293, Apr. 2009.

[2] M. Singh, A. Chakrapani, and D. O'Hagan, "Nanoparticles and microparticles as vaccine-delivery systems," Expert Rev Vaccines, vol. 6, pp. 797-808, Oct. 2007.

[3] S. Manmohan, Vaccine Adjuvants and Delivery Systems, Wiley Interscience, John Wiley \& Sons, Inc., Hoboken, New Jersey, 2007.

[4] J. C. Cox and A. R. Coulter, "Adjuvants-a classification and review of their mode of action," Vaccine, vol.15, pp. 248-256, Feb. 1997.

[5] C. Confavreux, A. Suissa, P. Saddier, V. Bourdes, and S. Vukusic, "Vaccinations and the risk of relapse of multiple sclerosis. Vaccines in Multiple Sclerosis Group," N. Engl. J. Med., pp. 319-326, Feb. 2001.

[6] L. Zhang, F. Gu, J. M. Chan, A. Z. Wang, and R. S. Langer, and O. C. Farokhzad, "Nanoparticles in medicine: therapeutic applications and developments," Clin. Pharmacol. Ther., vol. 83, pp. 761-769, May 2008.

[7] D. F. Emerich and C. G. Thanos "Targeted nanoparticle-based drug delivery and diagnosis," J. Drug. Target., vol. 15, pp. 163-183, Apr. 2007.

[8] M. Singh, J. R. Carlson, M. Briones, M. Ugozzoli, J. Kazzaz, J. Barackman, G. Ott, and D. O. Hogan, "A comparison of biodegradable microparticles and MF59 as systemic adjuvants for recombinant gD from HSV-2," Vaccine, vol. 16, pp. 1822-1827, Nov. 1998.

[9] R. K. Gupta, "Aluminium compounds as vaccine adjuvants," Adv Drug. Deliv. Rev., vol. 32, pp. 155-172, Jul. 1998

[10] E. B. Lindblad, "Aluminium adjuvants," in Theory and Practical Application of Adjuvants, D. E. S. Stewart-Tull, Ed., Wiley, Chichester., 1995, pp. 21-35.

[11] L. Stacy, W. Claus, M. Tulin, and J. Steve, "Calcium phosphate nanoparticle adjuvant," Clin.Diagn. Lab. Immunol., vol. 7, no. 6, pp. 899-903, Nov. 2000.

[12] C. Roduit, P. Bozzotti, N. Mielearek, H. Lambert, D. Guidice, C. Locht, and A. Siegrist, "Immunogenicity and protective efficacy of neonatal vaccination against Bordetella pertussis in a murine model: Evidence for early control of pertussis," Infect Immun., vol. 70, no. 7, pp. 3521-3528, Jul. 2002.

[13] H. Tamizifar, A. Robinson, R. Jennings, and C. Potter, "Immune response and protection against influenza $\mathrm{A}$ infection in mice immunized with subunit influenza A vaccine in combination with whole cell or acellular DTP vaccine," Vaccine, vol. 13, no. 16, pp. 1539-1546, Nov. 1995

[14] J. D. Bancroft and M. Gamble, Theory and Practice of Histological Techniques, $5^{\text {th }}$ Ed.. Churchill Livingstone, Edinburgh, London, 2002.

[15] N. Goto, H. Kato, and J. I. Maeyama, "Local tissue irritating effects and adjuvant activities of calcium phosphate and aluminum hydroxide with different physical properties," Vaccine, vol. 15, pp. 1364-1370, Aug-Sep. 1997.

[16] A. M. Gawish, A. M. Issa, A. M. Hassan, and Sh. Ramdan, "Morphometrical, histopathological, and cytogenetical ameliorating effects of green tea extract on nicotine toxicity of the testis of rats," $J$. Am. Sci., vol. 6, no. 11, pp. 401-411, Sep. 2010.

[17] M. F. Powell and M. J. Newman (Ed.), "Adjuvant properties of aluminum and calcium compounds," in Vaccine Design: The Subunit and Adjuvant Approach, Plenum Publishing Corp., New York, N. Y. pp. 229-248, 1995. 
[18] Q. He, A. R. Mitchell, S. L. Johnson, C. Wagner-Bartak, T. Morcol, and S. J. D. Bell, "Calcium phosphate nanoparticle adjuvant," Clin Diagn Lab Immunol., vol. 7, no. 6, pp. 899-903, Nov. 2000.

[19] J. L. Feldkamp, J. L. White, and S. L. Hem, "Effect of surface charge and particle size on gel structure of aluminium hydroxycarbonate gel," J. Pharm. Sci., vol. 71, pp. 43-46, Jan. 1982.

[20] M. R. Ickovic, E. H. Relyveld, and E. Henocq, "Calcium phosphate adjuvanted allergens: Total and specific IgE levels before and after immu-notherapy with house dust and Dermatophagoides pteronyssinus extracts," Ann. Immunol., (Paris), vol. 134D, pp. 385398, Nov.-Dec. 1983.

[21] M. A. K. Abdelhalim and B. Jarrar, "Histological alterations in the liver of rats induced by different gold nanoparticle sizes, doses and exposure duration," J. Nanobiotech, vol. 10, pp. 5-13, Jan. 2012,

[22] M. A. K. Abdelhalim and B. M. Jarrar, "Gold nanoparticles induced cloudy swelling to hydropic degeneration, cytoplasmic hyaline vacuolation, polymorphism, binucleation, karyopyknosis, karyolysis, karyorrhexis and necrosis in the liver," Lipids in Health and Disease, vol. 10, pp. 166-172, Sep. 2011.

[23] S. Segerer, P. J. Nelson, and D. Schlöndorff, "Chemokines, chemokine receptors, and renal disease: from basic science to pathophysiologic and therapeutic studies," J. Am. Soc. Nephrol., vol.11, pp.152-176, Jan. 2000.

[24] J. Savory and R. M. Garruto, "Aluminum, tau protein, and Alzheimer's disease: an important link?" Nutrition, vol. 14, pp. 313314, Mar. 1998.

[25] M. Vogelbruch, B. Nuss, M. Korner, A. Kapp, P. Kiehl, and W. Bohm, "Aluminium-induced granulomas after inaccurate intradermal hyposensitization injections of aluminium-adsorbed depot preparation," Allergy, vol. 55, pp. 883-887, Sep. 2000.

[26] K. Redhead, G. J. Quinlan, R. G. Das, and J. M. C. Gutteridge, "Aluminium-adjuvante vaccines transiently increase aluminium levels in murine brain tissue," Pharmacol. Toxicol., vol. 70, pp. 278280, Apr. 1992.

[27] B. Platt, G. Fiddler, G. Riedel, and Z. Henderson, "Aluminium toxicity in the rat brain:histochemical and immunocytochemical evidence," Brain. Res. Bull., vol. 55, pp. 257-267, May 2001.

[28] B. M. Barth, R. Sharma, E. I. Altinoglu, T. T. Morgan, S. S. Shanmugavelandy, J. M. Kaiser, C. McGovern, G. L. Matters, J. P. Smith, M. Kester, and J. H. Adair, "Bioconjugation of calcium phosphosilicate composite nanoparticles for selective targeting of human breast and pancreatic cancers in vivo," ACS Nano, vol. 4, no. 3, pp. 1279-1287, Mar. 2010.

[29] H. S. Muddana, T. T. Morgan, J. H. Adair, and P. J. Butler, "Photophysics of Cy3-encapsulated calcium phosphate nanoparticles," Nano Letters, vol. 4, pp.1559-1566, Apr. 2009.

[30] D. Grimm, K. L. Streetz, C. L. Jopling, T. A. Storm, K. Pandey, C. R. Davis, P. Marion, F. Salazar, and M. A. Kay, "Fatality in mice due to oversaturation of cellular microRNA/short hairpin RNA pathways," Nature, vol. 441, no. 7092, pp. 537-541, May 2006.

[31] M. Vogelbruch, B. Nuss, M. Körner, A. Kapp, P. Kiehl, and W. Bohm, "Aluminium-induced granulomas after inaccurate intradermal hyposensitization injections of aluminium-adsorbed depot preparations," Allergy, vol. 55, pp. 883-887, Sep. 2000.

[32] E. Bergfors, C. Bjorkelund, and B. Trollfors, "Nineteen cases of persistent pruritic nodules and contact allergy to aluminium after injection of commonly used aluminium-adsorbed vaccines," Eur. J, Pediatr., vol. 164, pp. 691-697, Nov. 2005.

[33] S. Tamuly and M. K. Saxena, "Preparation of calcium phosphate nanoparticles and evaluation of their effects on muscle cells of rat," Current Science, vol. 102, no. 4, pp. 610-612, Feb. 2012.

[34] N. Goto, H. Kato, and J. I. Maeyama, "Local tissue irritating effects and adjuvant activities of calcium phosphate and aluminum hydroxide with different physical properties," Vaccine, vol. 15, pp.1364-1370, Aug.-Sep.1997.

[35] E. Oberdorster, "Manufactured nanomaterials (fullerenes, C60) induce oxidative stress in the brain of juvenile largemouth bass," Environ.Health. Perspect., vol. 112, no. 10, pp. 1058-1062, Jul. 2004.

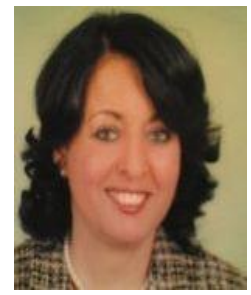

Aliaa Mahmoud Issa was born in Cairo, Egypt, April 1955. She got her B.Sc degree from Faculty of Science, Zoology Department, Cairo University in 1977. She was awarded her master degree from Cairo University. The Ph.D degree was awarded from Roswell Park Memorial Institute, Buffalo, USA and Cairo University in cancer studies.

She is a professor doctor of histology and histo-hemistry at the Zoology dept., Faculty of Science, Cairo university and Head of the Cell, Histology and Genetics section at the Zoology department. She teaches courses at the undergraduate and postgraduate levels. She supervises and evaluates Ph.d and master theses on different subjects such as hepatic cancer, sterility caused by gene mutation, stem cells... She is working with Quality Assurance unit at the faculty of science, Cairo University as Rapporteur of the Education portfolio of the Faculty. She is an external examiner at the Promotion Committee of Zoology for assoc. \& prof. positions degrees. Her research interests are cancer biology, application of nanoparticles, sterility and gene effects.

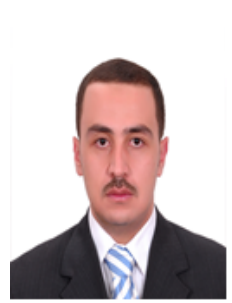

Mohamed Shaaban Mohamed Salim was born in Fayoum, Egypt, September 1982. He got his B.Sc degree from the Faculty of Science, Cairo University, Fayoum branch in 2005. He was awarded his master degree in 2013, from the Faculty of Science, Cairo University, Giza,

He started his Ph.D dissertation on Stem cells field. He works as the head of Sterile Area Section of the Holding company for Biological Products \& Vaccines - The Egyptian Company for production of vaccines, sera and drugs. He is supervising the production of many sterile biological products (vaccines \& sera) under aseptic processing following GMP (Good manufacturing procedures).

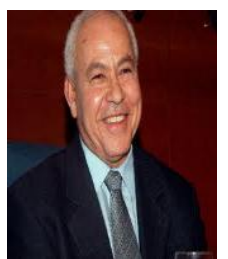

Hamdallah Zedan was born in Behara, Egypt in 1944. He is graduated from the Faculty of Pharmacy, Cairo University, in 1967. He got his Ph.D (Microbiology) from Montreal Universities, Canada $\mathrm{He}$ is a professor of microbiology and immunology, Faculty of Pharmacy, Cairo University and Chairman of the Pharmaceutical Studies Committee and Sector. $\mathrm{He}$ is also a supreme Council of Universities, Egypt. He supervised and directed research programs leading to M.Sc. and Ph.D. in Microbiology and Immunology (70 candidates) and published more than 100 papers. His fields of research are the microbial enzymes, radio-sterilization, microbial drug resistance, immobilized cell fermentation, mycotoxins, microbial contamination of pharmaceuticals recombinant DNA technology, vaccines and immunological products, biosafety and intellectual property rights (IPR). From 2007 to 2013, he was the Chairman of the Egyptian Company for Sera, Vaccines and Drugs. He held several positions with the United Nations including member of many scientific and advisory committees

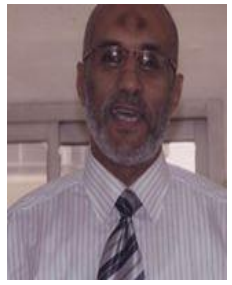

Aly Fahmy Mohamed was born in Cairo in 1955. He is graduated from the Faculty of Science in 1977. $\mathrm{He}$ is a general manager of Applied Research Sector at the Egyptian Company for biological products, Vaccines and Drugs (EgyVac). His research interests are on vaccine production and development, virus isolation and identification, and antiviral, anticancer researches. He published 44 papers $(15$ internationally published)

Abdel-Razik H. Farrag was born in Cairo in 1954. He is a professor of Pathology at the National Research Institute, Giza, Egypt. 